\title{
Interaction between endomycorrhizae and rhizosphere fungi in soils of Iraq
}

\author{
T. M. MUSHIN, J. N. NEMA
}

Department of Biology, College of Education, Basrah University Basrah, Iraq

$\mathrm{M}$ us h in T. M., $\mathrm{Nem}$ a J. N.: Interaction between endonycorrhizae and rhizosphere fungi in soils in Iraq. Acta Mycol. 32 (1): 41-50, 1997.

Endomycorrhizal and rhizospheric fungi were recovered from soil samples collected from the toot of plants in Iraq. The relations between the two fungal populations were investigated.

Key words: endomycorrhizac, rhizosphere fungi, fungi populations.

\section{INTRODUCTION}

The mutualistic association between plants and mycorrhizal fungi is beneficial. The main role of mycorrhizae is to supply the essential nutricional elements from soil to the plant roots and improving the growth of host plants ( $\mathrm{H}$ a $\mathrm{r}$ l e y 1989). In additions, mycorrhizae have an impact on the soil surrounding plant roots ( $\mathrm{n} \mathrm{g} \mathrm{h} \mathrm{a} \mathrm{m,} \mathrm{M} \mathrm{o} 1 \mathrm{i} \mathrm{n}$ a 1991). Nevertheless, the interaction between mycorrhizal fungi and soil microorganisms including bacteria, protozoa, nemaodes and pathogenic fungi have received considerable attention (S y I vi a 1990; I n g h a m, M o lin a 1991). Mycorrhizae protect plants from nematodes infection (K e $11 \mathrm{a} \mathrm{m}, \mathrm{S} \mathrm{che} \mathrm{n} \mathrm{c} \mathrm{k} \mathrm{1980;}$ $\mathrm{S} h$ a r m a J o h r i, G i a $\mathrm{n}$ i $\mathrm{n}$ a z z i 1992) affecting the bacterial populations in soil (A mes, R e id, I n g h m 1984) and reducing the pathogenicity of parastic fungi ( $\mathrm{Z}$ a m boli m, S c h e n c k 1993; G i ova n netti, A vio, S a 1 ut in i 1991). However, little information is available regarding the interaction between endomycorhizae and rhizospheric fungi in soil and only few cases have been reported (D a n i e ls, M e n g e 1980; S c he n c k 1981; K u ce y 1987).

In this study an attempt was made to demonstrate the relationship between endomycorrhizae and rhizospheric fungi in soils of five selected host plants in Iraq. 


\section{MATERIALS AND METHODS}

Soil samples were taken from root zones (from a depth of $10-15 \mathrm{~cm}$ ) of five host plant species, namely: Cordia myxia L., Ficus carica L., Lawsonia inermis L., Punica granatum L., and Vitis vinifera L. growing in Southern Iraq. A total of ninety soil samples were taken bimontly between January and November 1994. At each collecting time, $100 \mathrm{~g}$ soil sample per plant was placed in a plastic bag and brought to the laboratory for fungal spore isolation. Soil properties were determined such as: $\mathrm{pH}$ (6.6-7.5), conductivity (4.5-9.8 mmohs), moisture $(7.8-12.8 \%$ ) and soil texture (silt $60.1 \%$, clay 35.3 , sand 1.6 ).

Twenty grams of each soil sample were processed to recover spores of endomycorrhizal fungi using wet-sieving technique following $\mathrm{K}$ os $\mathrm{ke}$, $\mathrm{H}$ a 1 v e $\mathrm{r}$ s o $\mathrm{n}$ (1981). Spores enumeration was made according to G i ov a n n e t t i, M os s e (1980). Triplicates were used for each soil sample per collection. For isolation of rhizosphaeric fungi, the dilution plating method was conducted as described by W a r c u p (1950); 20 grams of soil were diluted in $100 \mathrm{ml}$ of distilled water and subsequently $1 \mathrm{ml}$ of soil suspension was transfered into a Petri-dish to which Czapek agar medium was added. Fungal colonies were surveyed and counted after $4-5$ days after being incubated at $20^{\circ} \mathrm{C}$. Triplicate were made for soil samples.

\section{RESULTS}

A total of 17 species of endomycorrhizal fungi recovered from soils collected from the five host plants (Table 1). The number of species found was as follows: 9 species in samples of Cordia myxia, 11 species with Ficus carica and with Vitis vinifera, 12 species with Lawsonia inermis and with Punica granatum. Out of them four taxa namely Acaulospora bireticulata, A. scrobiculata, A. trappei, Endogone incrassata and Glomus fasciculatum were common for all the plants. The total spore number of endomycorrhizae varied is in the collecting time and in the plants predominant species were Glomus fasciculatum, G. leptotichum, and Acaulospora laevis in the soils of the host plants (Table 1). Seasonal variations in spore number of endomycorrhizae was observed. High total spore number was detected in January-March for all the soil samples, except for Lawsonia inermis. A low total spore number was noted in September (Table 1). Among the host plants Cordia myxia rendered the highest spores number while Vitis vinifera showed the lowest spore number of endomycorrhizae.

The present data showed that 17 species of rhizospheric fungi inhabited in the soils of the plants studied (Table 2). Among the recovered fungi, 10 species were present in the soil sample of $C$. myxia, 12 species $-F$. carica, $13-P$. 
granatum and 14 species $-L$. inermis and $V$. vinifera. Among these taxa seven species, i.e., Alternaria alternata, Aspergillus fumigatus, A. niger. Cladosporium herbarum, Cunninghamella echinulata, Fusarium moniliforme (and Penicillium sp.) were common for the plant soils. The dominating fungi were: Aspergillus niger. Cladosporium herbarum (and Penicillium sp.) in all soil samples and through out the collecting period. Temporal variation in the total number of fungal isolates was observed (Table 2). The maximum number of isolates was encountered in September, except for Lawsonia inermis, and the lowest number of isolates was noted in March, except for Cordia myxia.

Data, regarding the total number of isolates of endomycorrhizae and rhizosphere fungi, were presented according to the dates of collection for each plant (Fig. 1). The general seasonal variation trend of endomycorrhizal fungi showed that the highest population density was in March and the lowest in September. A reverse relationship was observed for the rhizospheric fungal population, i.e. maximum population density was noted in September and minimum in March (Fig. 1).

\section{DISCUSSION}

The markedly varied species composition and population density of endomycorrhizal and rhizospheric fungi can be related to the nature of the host plants. It has been stated that host plants play a role in the presence or absence of endomycorrhizae, which is, in part, due to root exudation process (I n$\mathrm{g} \mathrm{h}$ a m, M ol in a 1991). Consequently, any changes in root exudation would affect the fungal colonization and their populations. The low density in the population of endomycorrhizae in September is possible associated with the low amount of root exudates produced by plants at the scenescent growth stage. These observations are in agreement with other studies ( ( $\mathrm{G} \mathrm{e} \mathrm{m} \mathrm{m} \mathrm{a,} \mathrm{K} \mathrm{o} \mathrm{s} \mathrm{k} \mathrm{e,}$ Carreiro 1989 ; M c G e e 1989 ; O ke ef, S y l vi a 1991 ; S y 1 vi a, W ill i a $\mathrm{m} \mathrm{s}$ 1992). These studies showed that high and low spore production by endomycorrhizae occur at early and late plant growth stages.

The present study also demonstrated that the endomycorrhizal association developed more frequently, e.g., in Glomus fueganum which was more abundant in the soil of the host C. myxia, Glomus fasciculatum in the soil of F. carica, Glomus aggregatum seemed to have a wider range of occurence in many plants. This is in agreement with the finding of $(\mathrm{H} \mathrm{e} \mathrm{r} \mathrm{i} \mathrm{c} \mathrm{k}, \mathrm{B} 1 \mathrm{oom}$ 1983; H e t r i c k, B 1 o o m 1986).

On the other hand, it appeared that soils of the selected plants harboring diverse taxa of rhizosphere fungi. Nevertheless, seasonal variations in rhizospheric fungal isolates can be related to different biotic and abiotic factors (P u g h 1980; D o m s ch et al. 1980; Ch r is ten s en 1989). The high 
$T$ i $b$ Mean spore number (per $10 \mathrm{~g}$ of soil) of endomycorrhizal fungal (ENMF)

\begin{tabular}{|c|c|c|c|c|c|c|c|c|c|c|c|c|}
\hline \multirow{2}{*}{ ENMF } & \multicolumn{6}{|c|}{ C. myxia } & \multicolumn{6}{|c|}{ L. inermis } \\
\hline & Jan. & Mar. & May & July & Sept. & Nov. & Jan. & Mar. & May & July & Sept. & Nov \\
\hline $\begin{array}{l}\text { Acaulospora biradiculata Roth. } \\
\text { et Trappe } \\
\text { - Iaevis Gerd. et Trappe } \\
\text { - scrobiculata Trappe } \\
\text { - digitata Walker et Trappe } \\
\text { - Irappei Ames et Linder }\end{array}$ & 14 & . & $\begin{array}{l}9 \\
9 \\
6\end{array}$ & 10 &  & $\begin{array}{l}10 \\
: \\
:\end{array}$ & $\begin{array}{l} \\
9 \\
5\end{array}$ & * & $\begin{array}{c}25 \\
13 \\
5 \\
5\end{array}$ & $\begin{array}{l}4 \\
8 \\
\end{array}$ & \begin{tabular}{c|}
5 \\
+ \\
15 \\
15
\end{tabular} & $\begin{array}{l}9 \\
7 \\
\end{array}$ \\
\hline $\begin{array}{l}\text { Glomus aggregatum Schenck } \\
\text { et Smith }\end{array}$ & 19 & 19 & 20 & 31 & $=$ & . & . & 10 & $=$ & 22 & 10 & + \\
\hline - albidum Walkers et Rhod. & $=$ & - & . & . & . & . & . & . & $=$ & 4 & . & . \\
\hline $\begin{array}{l}\text { - deserticola Trappe, } \\
\text { Bloss et Menge }\end{array}$ & 10 & . & 9 & = & 15 & 22 & . & $=$ & 15 & $=$ & + & 10 \\
\hline $\begin{array}{l}\text { - fasciculatum (Thaxter) } \\
\text { Gerd. et Trappe }\end{array}$ & 51. & 40 & 15 & . & . & 10 & 10 & 18 & . & . & 15 & 10 \\
\hline $\begin{array}{l}\text { - fragile (Berk. et Br.) } \\
\text { Trappe et Gerd. }\end{array}$ & = & $=$ & $=$ & . & $=$ & $=$ & . & $=$ & 8 & . & + & $=$ \\
\hline $\begin{array}{l}\text { - fuegianum (Speg.) } \\
\text { Trappe et Gerd. }\end{array}$ & = & 5 & 12 & 23 & 33 & 33 & . & . & 10 & $=$ & 5 & * \\
\hline $\begin{array}{l}\text { - fulvum (Berk. et Br.) } \\
\text { Trappe et Gerd. }\end{array}$ & = & . & . & . & . & $=$ & . & . & $=$ & . & . & . \\
\hline - leprotrichum Schnck & 23 & 6 & 15 & 14 & 8 & - & . & . & " & . & " & : \\
\hline $\begin{array}{l}\text { - microaggregatum Koske, } \\
\text { Gemma et Olexis }\end{array}$ & $=$ & " & . & $=$ & * & . & . & 16 & " & 30 & * & + \\
\hline $\begin{array}{l}\text { - mosseae (Nicol. et Gerd.) } \\
\text { Gerd. et Trappe }\end{array}$ & . & " & . & . & . & . & . & . & . & . & . & . \\
\hline - occultum Walker & - & 10 & 2 & $=$ & 14 & 6 & $=$ & - & 7 & $=$ & $\therefore$ & 3 \\
\hline $\begin{array}{l}\text { - reticulatum Bhatt. et } \\
\text { Mukerji }\end{array}$ & - & " & * & = & * & . & . & - & * & . & . & . \\
\hline \multirow{2}{*}{ Total spores } & 128 & 99 & 104 & 78 & 72 & 71 & 32 & 44 & 83 & 84 & 73 & 59 \\
\hline & \multicolumn{6}{|c|}{552} & \multicolumn{6}{|c|}{365} \\
\hline
\end{tabular}


I e 1

species of five host plants throughout the study period

\begin{tabular}{|c|c|c|c|c|c|c|c|c|c|c|c|c|c|c|c|c|c|}
\hline \multicolumn{6}{|c|}{ F carica } & \multicolumn{6}{|c|}{ P. granatum } & \multicolumn{6}{|c|}{$V$. vinifera } \\
\hline Jan. & Mar. & May & July & Sept: & Nov. & Jan. & Mar. & May & July & Sept. & Nov: & Jan. & Mar. & May & July & Sept: & Nov. \\
\hline 8 & . & . & . & 10 & . & 12 & . & 6 & 7 & 10 & * & 10 & 10 & . & 14 & . & 6 \\
\hline * & 8 & $=$ & 13 & * & 21 & 7 & 20 & 20 & 28 & . & 14 & $=$ & . & 13 & 10 & 9 & 13 \\
\hline * & 17 & 27 & * & 7 & 20 & 10 & 15 & 12 & $\cdot$ & - & 10 & . & 6 & 8 & 12 & . & 6 \\
\hline . & . & . & . & 8 & 9 & + & . & . & . & . & . & 3 & . & $*$ & . & 19 & 25 \\
\hline+ & 20 & 16 & 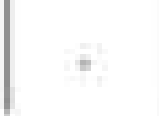 & + & . & - & $\cdot$ & 30 & 25 & . & + & . & . & 4 & . & . & 13 \\
\hline . & . & $=$ & . & . & . & . & · & . & . & . & . & 7 & . & 6 & 16 &. & - \\
\hline . & . & - & - & - & 8 & 10 & 28 & $=$ & . & . & - & - & . & . & . & . & . \\
\hline : & . & . & . & . & . & 8 & 4 & 7 & . & . & - & . & . & . & . & . & . \\
\hline 21 & 29 & 15 & 7 & ? & . & 18 & 21 & . & . & . & . & 17 & 28 & . & . & . & . \\
\hline 11 & . & . & 4 & . & . & 9 & * & . & . & . & . & . & + & . & . & . & . \\
\hline . & . & . & . & . & . & . & 2 & 4 & 15 & 13 & 15 & . & 2 & 13 & 11 & . & " \\
\hline 7 & 11 & $\Rightarrow$ & $=$ & $=$ & . & * & F & $\Rightarrow$ & $=$ & $=$ & $=$ & : & $\therefore$ & $\Rightarrow$ & . & $=$ & . \\
\hline 24 & 19 & 11 & $=$ & 21 & . & * & 10 & 8 & . & 31 & 14 & . & . & . & $=$ & . & " \\
\hline . & . & 1 & 12 & . & . & 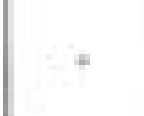 & $=$ & $\Rightarrow$ & . & $=$ & . & . & 4 & *. & 7 & 10 & . \\
\hline . & . & . & 4 & 16 & 10 & . & . & . & . & 10 & 21 & . & . & . & . & . & . \\
\hline . & . & . & . & . & . & * & 9 & 5 & . & . & . & 5 & 5 & 5 & . & . &. \\
\hline . & . & * & 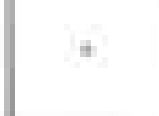 & . & . & 10 & . & . & 16 & 6 & . & 5 & . & 10 & . & 4 & 6 \\
\hline 71 & 104 & 70 & 40 & 62 & 68 & 84 & 94 & 92 & 103 & 70 & 74 & 46 & 55 & 59 & 70 & 42 & 69 \\
\hline & & 4. & 11 & & & & & 51 & 17 & & & & & & 39 & & \\
\hline
\end{tabular}


$\mathrm{T}$ a $\mathrm{b}$

Mean spore number (per $10 \mathrm{~g}$ of soil) of other rhizospheric fungal (ORF)

\begin{tabular}{|c|c|c|c|c|c|c|c|c|c|c|c|c|}
\hline \multirow{2}{*}{ ORF } & \multicolumn{6}{|c|}{ C. myxia } & \multicolumn{6}{|c|}{ L. inermis } \\
\hline & Jan. & Mar: & May & July & Sept: & Nov: & Jan. & Mar. & May & July & Sept. & Nov: \\
\hline Alternaria alternata (Fr.) Keissl. & 2 & . & 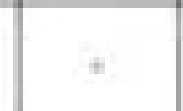 & + & - & $=$ & + & 9 & 6 & 3 & $*$ & ${ }^{*}$ \\
\hline Aspergillus fumigatus Fres. & " & 17 & $*$ & 6 & . & 5 & 30 & * & *. & 2 & + & 4 \\
\hline A. niger Tiegh. & + & $=$ & 22 & 58 & 10 & 42 & 79 & 15 & 17 & 45 & 40 & + \\
\hline A. terreus Thom & + & 3 & . & ; & 9 & + & + & 11 & $=$ & : & $\therefore$ & 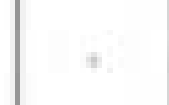 \\
\hline A. versicolor (Vuill.) Tirab & * & 4 & " & + & - & $=$ & 4 & + & + & : & $*$ & + \\
\hline $\begin{array}{l}\text { Cladosporium herbarum (Pers.) } \\
\text { Link }\end{array}$ & 1 & 3 & 6 & 10 & 69 & 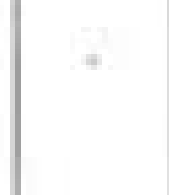 & 4 & + & 13 & i & 24 & 4 \\
\hline $\begin{array}{l}\text { Cumninghamella chinolata } \\
\text { Thaxter }\end{array}$ & 17 & $\cdot$ & 2 & 6 & 6 & $=$ & + & 4 & $=$ & 1 & + & + \\
\hline $\begin{array}{l}\text { Drechselara austeraliensis } \\
\text { Subram, Jain et Ellis }\end{array}$ & 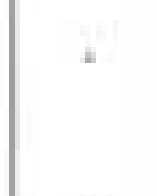 & s & i & i & $=$ & $\therefore$ & 6 & . & it & : & 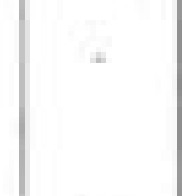 & + \\
\hline $\begin{array}{c}\text { (Drechselara sp. state of } \\
\text { Cochiobolws spici/er) }\end{array}$ & . & . & - & . & . & $=$ & . & 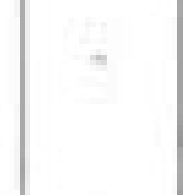 & * & , & . & ; \\
\hline Fusarium moniliforme Scheld. & - & $\therefore$ & * & : & - & 8 & , & . & . & : & . & 4 \\
\hline F. oxysporum Link & " & . & : & , & , & ? & * & : & $=$ & ? & . & r. \\
\hline Humicola grisea Traaen & " & $\rightarrow$ & + & : & $=$ & + & + & 10 & $=$ & + & 2 & 5 \\
\hline Mucor circinoid Wehmer & " & : & + & : & 4 & + & + & + & $=$ & : & 3 & + \\
\hline M. meahie Cooney et Emers. & $=$ & $\therefore$ & * & $=$ & $=$ & + & + & 3 & $=$ & 3 & $=$ & * \\
\hline Nigrospora oryzae (Berk.) Petch & . & . & * & . & " & + & + & . & - & : & . &  \\
\hline Paecillomyces roseum Bain. & + & 7 & $*$ & * & 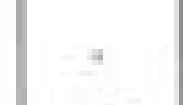 & + & 4 & $=$ & 25 & 21 & $=$ & + \\
\hline (Penicillimi sp.) & $=$ & 5 & 9 & $=$ & 7 & 64 & + & 50 & 10 & $=$ & 10 & 83 \\
\hline Stachybotrys atra Corda & $=$ & $\because$ & * & " & $=$ & $\Rightarrow$ & : & $=$ & $=$ & . & 1 & + \\
\hline Ulocladium botryitis Preuss & * & . & + & * & . & * & + & $=$ & . & . & . & * \\
\hline Total spores & 20 & 35 & 39 & 80 & 105 & 69 & 115 & 98 & 71 & 75 & 80 & 96 \\
\hline
\end{tabular}

number of fungal isolates in September accounted for the predominant species: Aspergillus niger, Cladosporium herbarum (and Penicillium sp.). This is perhaps due to the fact that there species compete strongly with other fungi species in soil (P u g h 1980).

Data presented in Fig. 1 showed a close reverse relationship between endomycorrhizal and rhizosphere fungal populations. The negative relationship was marked throughout the growing season and was related to the plants. It is evident that the low population density of rhizosphere fungi was influenced by the increase in endomycorrhizal population. Such inverse relationship might be the result of depletion in root exudates due to mycorrhizal colonization ( $\mathrm{L}$ a h e u r t e, B e r th e l in 1986), high competition of mycorrnizae with non-mycorrhizal fungi ( $\mathrm{G}$ r a h a m, M e n g e 1982), or to the production of antifungal substances produced by mycorrhizae ( $\mathrm{n} \mathrm{gh} \mathrm{a} \mathrm{m,} \mathrm{M} \mathrm{o} 1$ in a 1991) which may hinder the growth and populations of soilborn fungi. 
$1 \mathrm{e} 2$

species of five host plants

\begin{tabular}{|c|c|c|c|c|c|c|c|c|c|c|c|c|c|c|c|c|c|}
\hline \multicolumn{6}{|c|}{ F. carica } & \multicolumn{6}{|c|}{ P. granatum } & \multicolumn{6}{|c|}{$V$, vinifera } \\
\hline Jan. & Mar. & May & July & Sept. & Nov, & Jan. & Mar. & May & July & Sept. & Nov & Jan. & Mar. & May & July & Sept. & Nov. \\
\hline 2 & 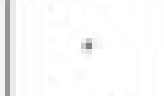 & . & $=$ & 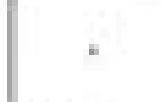 & 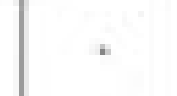 & 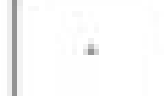 & + & 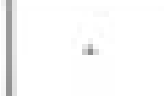 & . & 2 & $=$ & $=$ & 7 & 27 & 42 & 5 & 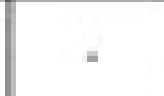 \\
\hline 2 & 7 & 10 & " & 11 & 4 & 22 & * & 30 & * & 3 & + & 14 & . & 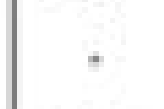 & & . & $=$ \\
\hline 21 & 16 & . & 17 & 24 & 27 & 54 & 34 & 18 & 55 & 77 & 41 & . & 50 & 58 & 47 & 30 & 49 \\
\hline . & . & = & 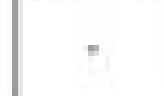 & . & . & . & : & . & . & . & $=$ & . & . & 8 & 3 & 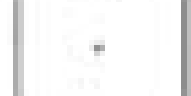 & = \\
\hline . & . & 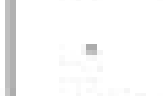 & " & " & . & . & . & . & . & . & . & , & . & 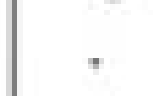 & . & F & 9 \\
\hline 49 & . & 78 & 76 & 37 & 50 & 32 & 20 & 17 & 24 & = & 8 & 41 & 13 & 22 & 15 & 60 & 30 \\
\hline - & 5 & 10 & 8 & 9 & " & 2 & 3 & 1 & 6 & . & . & 10 & + & + & 5 & " & " \\
\hline " & . & " & . & " & - & - & * & * & - & - & 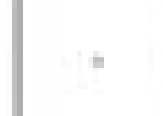 & . & + & * & - & - & " \\
\hline . & . & . & $=$ & , & . & . & . &  & . & $=$ & . & . & * & + & - & 2 & $=$ \\
\hline = & . & . & . & - & 4 & + & * & 2 & - & - & 2 & . & . & : & . & - & 4 \\
\hline " & . & . & " & 2 & . & . & * & * & * & 3 & . & . & . & + & . & 2 & 3 \\
\hline . & . &. & . & 4 & . & 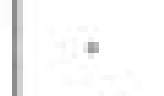 & . & . & . & 3 & . & . & 2 & . & . & 3 & . \\
\hline 16 & . & $\cdot$ & . & - & . & 12 & . & = & . & - & 11 & 20 & - & " & . & 8 & . \\
\hline . & 1 & . & . & . & . & . & . & . & . & . &. & 1 & 2 & . & " & . & " \\
\hline . & . & . & . & . & . & . & . & 3 & . & . & . & . & . & . & . & . & + \\
\hline . & . & . & . & . & 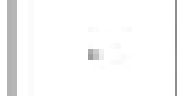 & . & " & $=$ & . & . &  & . & . & . & . & . & . \\
\hline$=$ & 10 & 4 & 10 & 13 & 12 & . & 12 & 16 & 13 & 27 & 31 & 79 & 55 & 37 & 31 & 83 & 15 \\
\hline . & . & . & $=$ & = & . & $=$ & $=$ & $=$ & $=$ & 2 & $=$ & . & . & . & $=$ & . & * \\
\hline . & . & . &. & 4 & 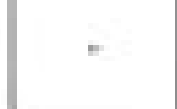 &  & . & . & $=$ & 4 & $\cdot$ & + & + & + & . & - & . \\
\hline 90 & 39 & 98 & 111 & 104 & 97 & 92 & 69 & 87 & 98 & 121 & 103 & 165 & 129 & 152 & 143 & 193 & 110 \\
\hline
\end{tabular}

The present results indicate that the endomycorrhizae infested soilborn inhibit or reduce the activity of rhizosphere fungi. Few reports concerning the relationship between endomycorrhizae and soilborn fungi are available and only single examples were cited in literature. D a n i e $1 \mathrm{~s}$ and $\mathrm{M}$ e n g e (1980) reported a negative interaction between Glomus macrocarpum and Pythium $\mathrm{sp}$. in soil of wheat. S c h e n c k and K e ll a m (1978) indicated that mycorrhizae reduced fungal pathogen attack on some plant crops. In addition, reduction of the soybean root-infecting fungi including Fusarium solani and Rhizoctonia solani was observed by $\mathrm{Z} \mathrm{a} \mathrm{m} \mathrm{b} \mathrm{o} \mathrm{i} \mathrm{m}$ and $\mathrm{S} \mathrm{ch} \mathrm{e} \mathrm{n} \mathrm{ck}$ (1983).

It is worth mentioning that among the isolated fungi which are known as common saprophytes, some species, however, were reported as plant pathogens (D o m s c h et al. 1980). These observations should lead to further studies regarding the introduction of endomycorrhizae into soil to reduce the negative effect of endomycorrhizae into soil to reduce the negative effect of other fungi. 




L. inermis
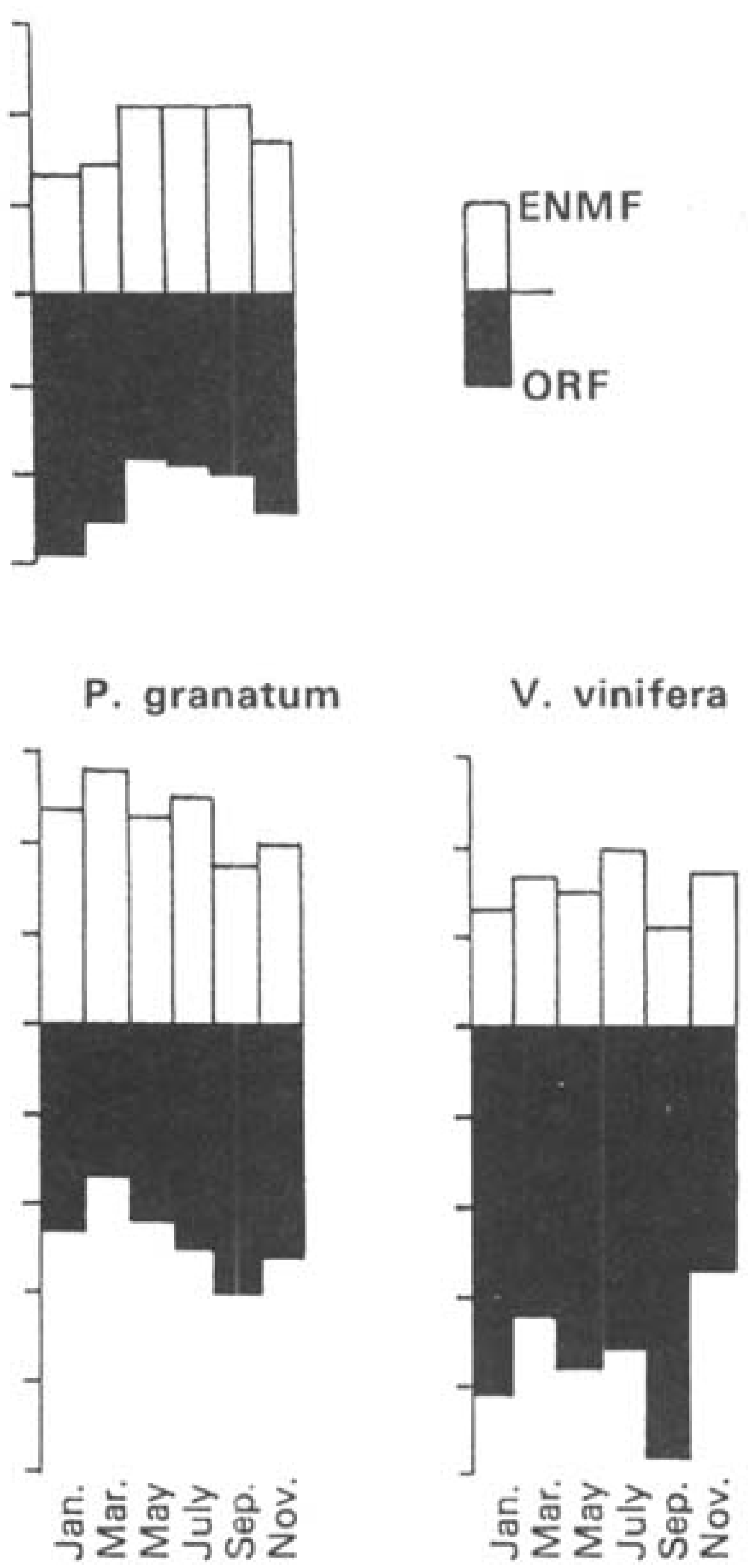

Fig. 1. Correlation between the total number of endomycorrhizal fungal (ENMF) spores and the total number of other rhizospheric fungal (ORF) spores in soil of five host plant species 


\section{REFERENCES}

A m es R. N, R e id C. P., I n g h a m E. R. 1984. Rhizosphere bacterial population responses to root colonization by a vesicular-arbuscular mycorrhizal fungus. New Phytol. 69: 555-563.

Christensen M., 1989. A view of fungal ecology. Mycologia 81: 1-19.

D a $\mathrm{n}$ i e Is B. A. M e n g e J. A. 1980. Hyperparasitism of vesicular-arbuscular mycorrhizal fungi. Phytopathology 70: $584-588$.

Dom se h K. H, G a m s W, A nde $\mathrm{s} s$ o n T. H. 1980. Compendium of soil fungi. Acad. Press, London.

G e m m a J. N., R os k e R. E., C a r r e i r o M. 1989 Seasonal dynamics of selected species of VA mycorrhizal fungi in a sand dune. Mycol. Res. 92: 317-321.

G i o va n e t t i M ., M oss e B. 1980. Evaluation of techniques for measuring vesicular-arbuscular mycorrhizal infection in roots. New Phytol. 84: 489-500.

G i ovannetti M., A vio I, Sa I u i in i L. 1991. Morphological, cytochemical and antogenetic characteristics of a new species of vesicular-arbuscular mycorrhizal fungi. Canad. J. Bot, 69: 161-167.

G r a h a m J. $\mathrm{H}_{44} \mathrm{M}$ e n g e J. A. 1982. Influence of vesicular-arbuscular mycorrhizac and soil phosphorus on take all disease of wheat. Phytopathology 72: 95-98.

$\mathrm{H}$ a $\mathrm{r}$ I e y J. L. 1989. The significance of mycorrhizae. Mycol. Res. 92: 129-139.

H e t i i c k B.A. D., B $100 \mathrm{~m}$ J. 1983. Vesicular-arbusvular mycorrhizac with native tall grass pratric and cultivated winter wheat. Canad. J. Bot. $61 \div 2140-2146$.

Hetrick B. A. D. B 1 o o m J. 1986. The influence of host plant on production and colonization ability of VAM spores. Mycologia 78: 32.

I $\mathrm{ng}$ h a m R. E., Mo i I in a $\mathrm{R}$. 1991. Interaction among mycorrnizal fungi, rhizosphere organisms, and plants. In: Barbosa P., Krischik V., Jones C.G. (Eds.). Microbial mediation of plant-herbivor interactions. 169-197.

Kellam M. K., S che n ck N. C. 1980. Interaction between a vesicular-arbuscular mycorrhiza fungus and root knote nematode on soybean. Phytopathology 70: 293-296.

K o s k e R. E., H a I vers o n W. L. 1981. Ecological studies of VAM in barrier sand dunes. Canad. J. Bot. 59: 1413-1422.

K u c e y R. M. N. 1987. Increases phosphrous uptake by wheat and field beans inoculated with a phosphorous - solubilizing Penicillim bilaji strain and with vesicular-arbuscular mycorrhizal fungi. Appl. Environ, Microbiol. 55: 2699-2703.

$\mathrm{L}$ a h e u i t e F. B e r the 1 in J. 1986. Influence of endomycorrhizal infection by Glomus mosseae on root exudation by maize. In: Physiology and genetical aspects of mycorrhizae. Eds.: by Gianinnazzi Pearson $V_{\text {tp }}$ Gianinazzi S. Instit. Nation. Res. Agron. 426-429.

M c g e e P. A. 1989. Variation in propagule numbers of vesicular arbuscular mycorrhizal fungi in semi arid soil. Mycol. Res. 92: 28-33.

O k e ef D. M., S y l v i a D. M. 1991. Mechanism of the vesicular arbuscular mycorrhizal plant growth response. In: Arora D.K., Mykerji K.G., Kundsen G.R. Hand book of applied mycology: Dekker M, New York.

$\mathrm{Pugh}$ G. J. F, 1980. Strategies in fungal ecology. Trans. Brit. Mycol. Soc. 75: 1-14.

S chenck N. C. 1981. Can mycorrhizae control root disease. Plant Disease 65: 230-234.

$\mathrm{S}$ c h e n ck N. C., K e 11 a m M. K. 1978. The influence of vesicular-arbuscular mycorrhizacoon disease development. Fla. Agric. Exp. Stn. Tech. Bull. 798: 16.

Sharma A. K., Johri B. N., G i a n i n a z z i S. 1992. Vesicular-arbuscular mycorrhizae in relation to plant disease. World J. Microbiol. Biotech. 8; 559-583.

S y I via D.M. 1990. Distribution, structure and function of external hyphae of vesicular- 
-arbuscular mycorrhizal fungi. In: Box J.E., Hammond L.H. (Eds.). Rhizosphere dynamics: 144-167. Westview Press, USA.

S y 1 vi a D. M., W i 11 i a m s S. E. 1992. Vesicular-arbuscular mycorrhizae and environmental

stress. In: Mycorrhizae in sustainable agriculture. Amer. Soc. Agron. 54: 101-124.

W a r c u p J. H. 1950. The soil plate method for isolation of fungi from soil. Nature, 166: 117-118. $\mathrm{Z}$ a m b o l i m L., S c h e n c k N. C. 1983. Reduction of the effects of pathogenic root infecting fungi on soybean by mycorrhizal fungi. Phytopathology 93: 1402-1405.

\section{Interakcje grzybów mikoryzowych i ryzosferowych w glebach Iraku}

Streszezenie

W próbkach gleby pobranej ze strefy korzeni pięciu roślin w Pld. Iraku wykryto obecność dziewiętnastu gatunków grzybów mikoryzowych i ryzosferowych. Największą liczbę endomikoryz stwierdzono w marcu, najmniejszą we wrześniu; odwrotny stosunek wystapil w przypadku izolatỏ grzybów ryzosferowych. W pierwszym przypadku dominowaty Glomus fasciculatum, G. leptotrichum i Acaulospora laevis, natomiast w drugim w glebie najwięcej bylo Aspergillus niger. Cladosporium herbarum oraz Penicillium sp. Przyczyną tak glębokich róznic pomiędzy tymi dwoma populacjami moglaby być konkurencja między nimi, zasiedlanie korzeni przez gatunki oddziaływujące niekorzystnie na grzyby i nie-mikoryzowe lub też przez wytwarzanie przez grzyby mikoryzowe substancji hamujących lub redukujących populacje gatunków ryzosferowych. 